\title{
Improving effect of a probiotic mixture on memory and learning abilities in D-galactose-treated aging mice
}

\author{
Shang-Tse Ho, ${ }^{1,2}$ Yueh-Tung Hsieh, ${ }^{1}$ Sheng-Yao Wang, ${ }^{1}$ and Ming-Ju Chen ${ }^{1 *}$ \\ ${ }^{1}$ Department of Animal Science and Technology, National Taiwan University, Taipei 10617, Taiwan \\ ${ }^{2}$ Agricultural Biotechnology Research Center, Academia Sinica, Taipei 11529, Taiwan
}

\begin{abstract}
The aim of this study was to evaluate the antiaging effect of a probiotic mixture using an in vivo mouse model in which aging was induced with D-galactose. Results of the Morris water maze test indicated that long-term administration of the probiotic mixture improved memory and learning abilities and ameliorated the apoptosis pattern in the hippocampus of aging mice treated with D-galactose. An antioxidation experiment indicated that administration of the probiotic mixture could restore activities of the antioxidant enzymes superoxide dismutase and catalase and inhibit the production of malondialdehyde. The antioxidant-related proteins nuclear factor erythroid 2-related factor 2 (Nrf2) and heme oxygenase-1 (HO-1) were upregulated in liver after treatment of D-galactose-treated aging mice with probiotics. Finally, the probiotic treatment did affect the production of short-chain fatty acids in D-galactose-treated aging mice. Our results highlighted a possible antioxidative effect triggered by short-chain fatty acids that contributed to improving the memory and learning abilities following treatment with the probiotic mixture and suggested that probiotics could serve as a therapy to modulate physiological function. Key words: aging, probiotic mixture, reactive oxygen species (ROS), antioxidant
\end{abstract}

\section{INTRODUCTION}

With an increase in the global population and extension of lifespan, aging is becoming one of the most important public health issues (Chen et al., 2017). Aging is a known major risk factor in many human diseases such as cancer, idiopathic Parkinson's disease, type I and II diabetes, cardiovascular disease, and metabolic syndrome (Barzilai et al., 2012; Dillin et al., 2014). In addition, obvious dysfunctions of cognitive, memory,

Received October 9, 2018

Accepted November 13, 2018

*Corresponding author: cmj@ntu.edu.tw and learning abilities, as well as brain lesions, are observed during the aging process. Because aging-related diseases significantly affect human life, there is great interest in understanding, preventing, and treating abnormal aging processes. Previous studies reported that mitochondrial reactive oxygen species (ROS) and free radicals play critical roles in cellular oxidative damage and aging (Barja, 2014; Rottenberg and Hoek, 2017). The free-radical theory of aging, a widely accepted concept, states that mutations of the mitochondrial DNA accumulate with increasing age and lead to a loss of function with subsequent acceleration of cell death (Ward et al., 2014; Raz and Daugherty, 2018). Thus, free radical damage associated with environmental conditions and free radicals produced by the inherent aging process are crucial factors involved in this theory (Harman, 2002). Further evidence has shown that longterm exposure of D-galactose induces aging through formation of ROS and elevation of oxidative stress in mice (Zhong et al., 2009; Parameshwaran et al., 2010). Decreasing ROS and free radical formation might provide a possible solution for aging-related disorders.

In the past decade, antioxidant activity of lactic acid bacteria (LAB) and LAB-related products have been frequently reported (Li et al., 2012; Tang et al., 2016). Many methods are used to evaluate antioxidant properties of a test sample. Radical scavenging activity [e.g., 1,1-diphenyl-2-picrylhydrazyl (DPPH) radical, superoxide anion, oxygen radical], hydrogen peroxide scavenging activity, $\mathrm{Fe}^{2+}$ chelating activity, reducing power, and inhibitory effect of lipid peroxidation are common protocols for in vitro antioxidant assays (Alam et al., 2013). These assay systems are mainly focused on changes in antioxidant enzyme and biochemical parameters, including glutathione peroxidase, superoxide dismutase (SOD), and malondialdehyde (MDA) expression of experimental animals after treatment with testing samples (Alam et al., 2013). Several LAB strains have been reported to have antiaging effects that might be due to their radical scavenging activity and enhancement effect of antioxidant enzymes (Yu et al., 2016; Zhao et al., 2017). 
Table 1. Treatment groups of D-galactose-induced aging mouse model

\begin{tabular}{lll}
\hline Group & \multicolumn{1}{c}{ Administration ${ }^{1}$} & Treatment $^{2}$ \\
\hline Control & Saline & PBS \\
Negative control & Saline & $100 \mathrm{mg} / \mathrm{kg}$ of BW of D-galactose \\
$\mathrm{PM}-1^{3}$ & $1 \times 10^{8} \mathrm{cfu}$ of PM-1/d & $100 \mathrm{mg} / \mathrm{kg}$ of BW of D-galactose \\
${ }^{1}$ Oral administration. \\
${ }^{2}$ Subcutaneous injection of D-galactose to induce aging. \\
${ }^{3}$ Probiotic mixture-1, containing Lactobacillus paracasei ssp. paracasei BCRC 12188, Lactobacillus plantarum \\
BCRC 12251, and Streptococcus thermophilus BCRC 13869.
\end{tabular}

In our previous study, we found that a probiotic mixture (PM-1, containing Lactobacillus paracasei ssp. paracasei BCRC 12188, Lactobacillus plantarum BCRC 12251, and Streptococcus thermophilus BCRC13869) exhibited a uremic toxin-reducing effect in a cisplatininduced nephrotoxicity rodent model. Because cisplatin is a strong oxidant agent, one of the mechanisms involved in the uremic toxin-reducing effect of PM-1 might be its antioxidant activity. Thus, we hypothesized that PM-1 possesses antiaging effects. To test our hypothesis, we evaluated the protective effect of PM-1, and related mechanisms, on a D-galactose-induced aging mouse model.

\section{MATERIALS AND METHODS}

\section{Lactic Acid Bacterial Sample Preparation and Identification}

Lactobacillus paracasei ssp. paracasei BCRC 12188, L. plantarum BCRC 12251, and S. thermophilus BCRC13869 were purchased from Bioresource Collection and Research Center (BCRC, Hsinchu, Taiwan). The LAB strains were cultured in Lactobacilli de Man, Rogosa, and Sharpe (Acumedia, Lansing, MI) at $37^{\circ} \mathrm{C}$ and harvested in log phase (optical density value $=0.8$ ) by washing and resuspending 3 times in PBS (Hyclone, South Logan, UT). After washing, the LAB cells were resuspended in $0.85 \%$ saline and adjusted to a concentration of $1 \times 10^{9} \mathrm{cfu} / \mathrm{mL}$ for the antioxidant activity assay.

\section{Animals and Experimental Design}

Ten-week-old C57B/CL6 mice (male) were purchased from BioLasco Taiwan Co. Ltd. (Ilan, Taiwan), and housed under $22 \pm 2^{\circ} \mathrm{C}, 40$ to $60 \%$ relative humidity, and a 12-h dark:light cycle. Before the experimental period, mice were acclimated to the environment for $7 \mathrm{~d}$. Mice were randomly divided into 3 groups $(\mathrm{n}=5)$ : control (no D-galactose, administered saline), negative control (D-galactose-induced aging, administered saline), and PM-1 group (D-galactose-induced aging, administered probiotic mixture), as shown in Table 1. Briefly, the experimental period of this study was $12 \mathrm{wk}$. The mice of the control group were daily administrated saline orally and treated with PBS by subcutaneous injection; the mice of the negative control group were daily administrated with saline and treated with $100 \mathrm{mg} / \mathrm{kg}$ of BW of D-galactose; the mice of the PM-1 group were daily administrated $1 \times 10^{8} \mathrm{cfu}$ of PM- 1 and treated with $100 \mathrm{mg} / \mathrm{kg}$ of BW of D-galactose. During the experimental period, mice received a laboratory rodent diet (LabDiet 5001, PMI Nutrition International LLC, Brentwood, MO) and water ad libitum. All procedures in the study were performed following the requirements of Institutional Animal Care and Use Committee (IACUC) of National Taiwan University.

\section{Morris Water Maze}

On d 21 and 70 of the PM-1 treatment, the Morris water maze was used to conduct the reference memory task, probe test, and working memory task for evaluation of long-term memory, spatial learning and memory, and short-term memory abilities of mice, respectively, as previously described (Chen et al., 2016a; Qu et al., 2016) with slight modifications. Briefly, the test was performed in a circular water pool (diameter: $120 \mathrm{~cm}$; height: $45 \mathrm{~cm}$ ). The pool was divided into 4 quadrants (I, II, III, and IV) and the water kept at room temperature $\left(22 \pm 2^{\circ} \mathrm{C}\right)$. The swimming orbits and times of test mice were monitored using an overhead camera during experimental period, and the data were analyzed using EthoVision XT 7 software (Noldus Information Technology, Wageningen, the Netherlands).

\section{Reference Memory Task}

This experiment was performed on d 22 to 24 (wk 3) and d 71 to 73 (wk 10) of the PM-1 treatment. The escape platform was placed in quadrant III of the pool. Mice were put into the water pool at randomly chosen start positions with their heads facing outside. Each mouse performed 4 trials per day, and the experimental duration was $90 \mathrm{~s}$. If mice could not find the 
escape platform within $90 \mathrm{~s}$, they were placed onto the platform for $15 \mathrm{~s}$ for guiding. All data were analyzed using EthoVision XT 7 software (Noldus Information Technology).

\section{Probe Test}

On d 24 and 73, the probe test was performed following the reference memory task. In brief, after removing the escape platform, the mice were put into quadrant I of the pool to perform this experiment for $90 \mathrm{~s}$. The staying time of mice in quadrant III (the previous location of the escape platform) was recorded to investigate the memory and learning ability of mice.

\section{Working Memory Task}

On d 25 to 27 and d 74 to 76 , the working memory task was performed to evaluate short-term memory. The escape platform was placed in different locations (quadrant I, II, or III) of the pool during the experimental period. Each mouse performed 5 trials per day. The first trial of each mouse in every day was the training procedure; only the results of trials 2 to 5 were recorded. The experimental duration was $90 \mathrm{~s}$, and the mice were placed onto platform for $15 \mathrm{~s}$ for guiding if they could not find the escape platform within $90 \mathrm{~s}$.

\section{Preparation of Tissues and Blood}

Mice were fasted overnight before being killed using $\mathrm{CO}_{2}$ at the end of this experiment (wk 12). Blood and tissues were collected after killing and plasma samples were collected by centrifugation at $1,100 \times g$ for 30 min. The organs were divided into 2 equal parts; one part was used for biochemical analysis and the other was immediately fixed in $10 \%$ formalin for histological analysis. All of the collected samples were stored at $-80^{\circ} \mathrm{C}$ freezer until analyzed.

\section{Detection of Antioxidant Biochemical Parameters}

The activities of antioxidant enzymes SOD and catalase (CAT) and lipid peroxidation level (measured as thiobarbituric acid reactive substances, TBARS) of test samples were determined according the manufacturer's instructions (SOD and CAT kit, TBARS kit; Cayman Chemical Co., Ann Arbor, MI).

\section{Immunohistochemistry Staining}

Brain tissue was obtained from healthy mice and D-galactose-treated aging mice to detect expression of the cleaved caspase- 3 protein. The tissue slides were deparaffinized and rehydrated in a Coplin jar by the following washing procedure: xylene for 5 min twice, $95 \%$ ethanol for $5 \mathrm{~min}, 70 \%$ ethanol for $5 \mathrm{~min}$, and deionized water for $5 \mathrm{~min}$. The immunohistochemistry staining was performed according to the manufacturer's protocol (Ultravision Detection System, Thermo Fisher Scientific, Waltham, MA).

\section{Western Blot Analysis}

Fifty milligrams of liver tissue was lysed in $100 \mu \mathrm{L}$ of radioimmunoprecipitation assay (RIPA) buffer containing $10 \%$ proteinase inhibitor and $10 \%$ phosphatase inhibitor (Sigma-Aldrich, St. Louis, MO) and boiled in a water bath at $100^{\circ} \mathrm{C}$ for $10 \mathrm{~min}$. Protein concentrations were measured by the bicinchoninic acid (BCA) Protein Assay kit (Thermo Fisher Scientific). Proteins $(40 \mu \mathrm{g})$ were loaded onto $12 \%$ SDS-PAGE gels for electrophoresis. After $4.5 \mathrm{~h}$ of electrophoresis, proteins were transferred to a polyvinylidene fluoride (PVDF) membrane and blocked (5\% skim milk in TBS-Tween buffer) for $1 \mathrm{~h}$. The membranes were incubated with primary antibodies for nuclear factor erythroid 2-related factor 2 (Nrf2), heme oxygenase-1 (HO-1), and $\beta$-actin (Abcam, Cambridge, MA) at a dilution rate of 1:1,000 in TBS-Tween buffer in $4^{\circ} \mathrm{C}$ overnight. Then, the blots were washed 3 times with TBS-Tween buffer, and incubated with horseradish peroxidase (HRP)-labeled rabbit secondary antibodies (Jackson ImmunoResearch Laboratories, West Grove, PA) for $2 \mathrm{~h}$. The signals of the blots were then developed using an enhanced chemiluminescence solution (Perkin-Elmer, Waltham, MA) and analyzed using the ChemiDoc Touch Imaging System (Bio-Rad, Hercules, CA).

\section{Quantitation of Short-Chain Fatty Acids Contents in Feces Sample}

Fecal samples were collected at the end of experiment. Feces $(0.01 \mathrm{~g})$ was extracted with $1 \mathrm{~mL}$ of $70 \%$ ethanol and the feces extract was then derivatized according to the method reported previously (Torii et al., 2010). The obtained fatty acid hydrazides were analyzed by HPLC using a PU-2089 pump (Jasco, Tokyo, Japan) equipped with an UV-4075 detector (Jasco; measurement wavelength $=400 \mathrm{~nm}$ ) and a 5- $\mu \mathrm{m}$ ReproSil $100 \mathrm{C} 18$ column $(250 \times 4.6$ mm i.d.; Dr. Maisch GmbH HPLC, Ammerbuch, Germany), at a column oven temperature of $50^{\circ} \mathrm{C}$. Acetonitrile:methanol:water (30:16:54, vol $/ \mathrm{vol} /$ vol; $\mathrm{pH}$ adjusted to 4.5 using $0.1 \%$ trifluoroacetic acid) was used as an isocratic mobile phase at a flow rate of $1.1 \mathrm{~mL} / \mathrm{min}$. 


\section{Statistical Analysis}

The results are expressed as means $\pm \operatorname{SEM}(n=5)$. Statistical analysis was performed with Student's $t$-test; a $P<0.05$ was considered statistically significant.

\section{RESULTS}

\section{Memory and Learning Activities After Administration of PM-1}

To investigate the effects of PM-1 on memory impairment in D-galactose-treated aging mice, we applied the Morris water maze test. After a 3-wk administration of PM-1, the performance of mice in the different treatment groups was evaluated and results are shown in Figure $1(\mathrm{~A}-\mathrm{C})$. Short-term administration of PM-1 did not have a significant effect on the reference memory task. Similarly, the results of probe test and working memory task revealed that short-term administration of PM-1 did not enhance memory and learning activities of D-galactose-treated aging mice.

The results of long-term (10 wk) administration of PM-1 on memory and learning activities of D-galactosetreated aging mice are shown in Figure $1(\mathrm{D}-\mathrm{F})$. For the reference memory task, the results indicated that mice in the PM-1 group exhibited better reference memory activity $(P<0.05)$ than those in the negative control group on the second and third days of the test (Figure 1D). As shown in Figure 1E, the cumulative duration (the time mice were in the target quadrant in the maze) of mice in the negative control group was significantly lower than that of mice in the control group $(P<0.05)$. The PM-1 treatment prolonged the cumulative duration of D-galactose-treated aging mice. Additionally, mice in the PM-1 group showed better working memory activity than mice in the negative control group. These results revealed that long-term administration of PM-1 significantly $(P<0.05)$ reversed the decline in memory and learning activities of D-galactose-treated aging mice.

\section{Protective Effect of PM-1 on Hippocampus Damage}

We further evaluated the protective effect of PM-1 on hippocampus damage of mice by immunohistochemical staining for cleaved caspase-3, an indicator of apoptosis and cell damage. The control group showed weak expression of cleaved caspase-3 in the hippocampus, whereas the negative control group showed strong expression of cleaved caspase-3. The PM-1 treatment sig-

Week 3

(A)

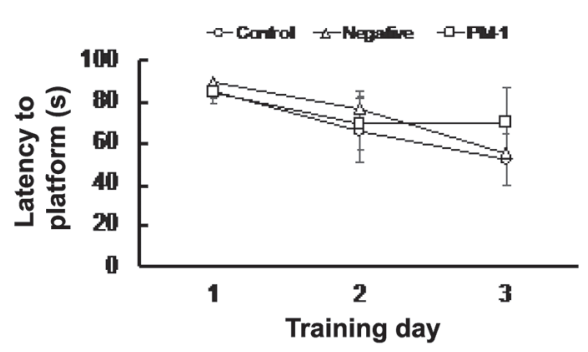

(B)

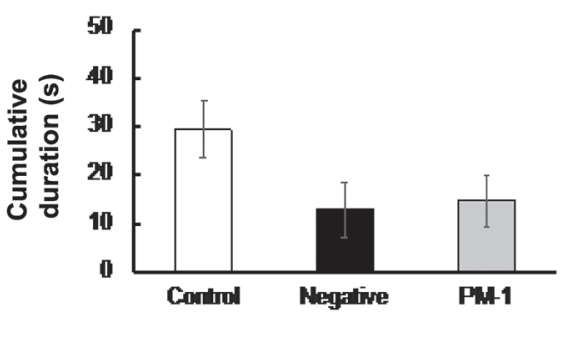

(C)

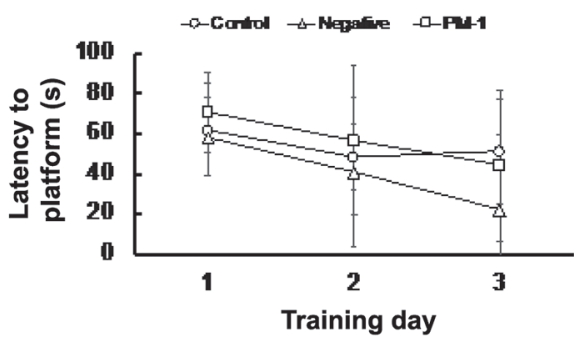

Week 10

(D)

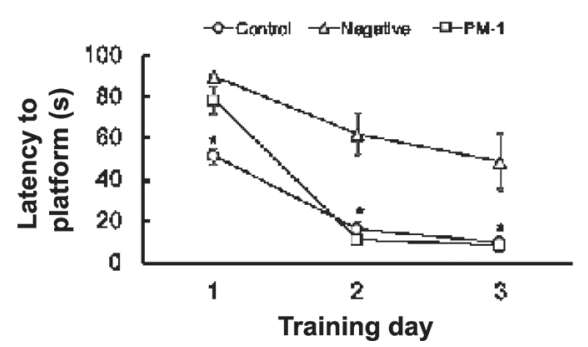

(E)

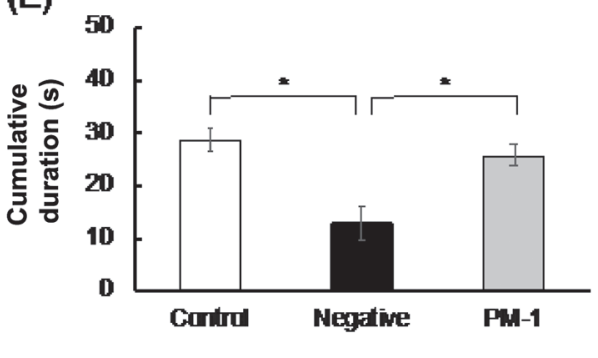

(F)

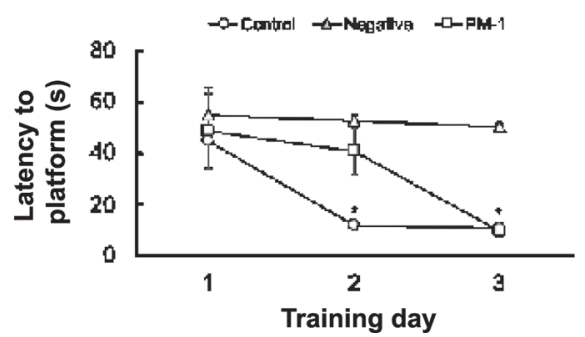

Figure 1. Performance in Morris water maze test for (A, D) reference memory task, (B, E) probe test, and (C, F) working memory task at wk $3(\mathrm{~A}, \mathrm{~B}, \mathrm{C})$ and wk $10(\mathrm{D}, \mathrm{E}, \mathrm{F})$. Values are the mean $\pm \mathrm{SEM}(\mathrm{n}=5)$. *Significantly different $(P<0.05)$ compared with the negative control group. Control, negative control, and probiotic mixture-1 (PM-1) treatments are as described in Table 1. 
(A)
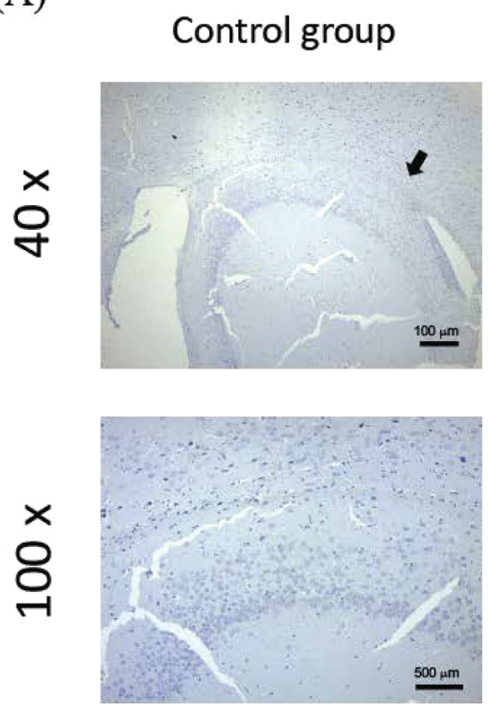

Negative control
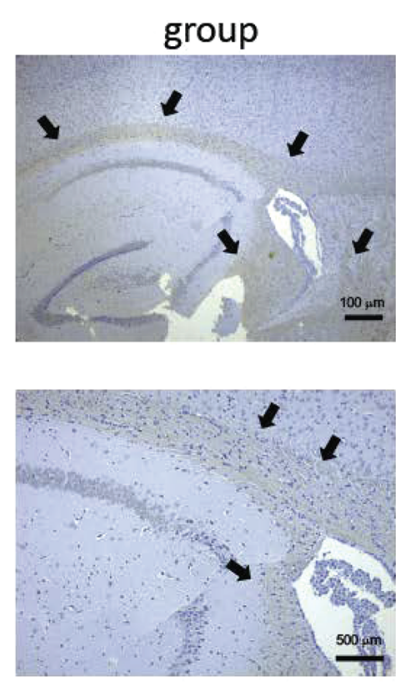

PM-1 group
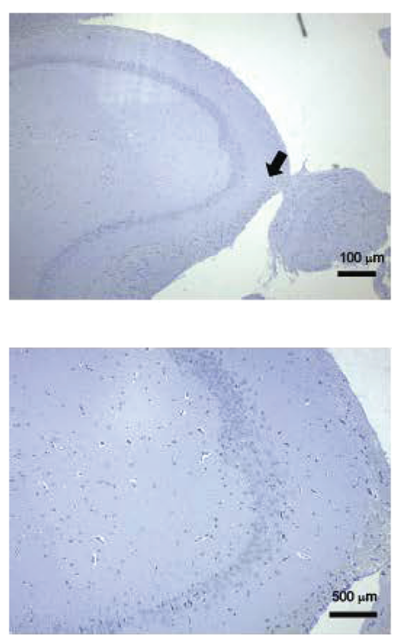

(B)

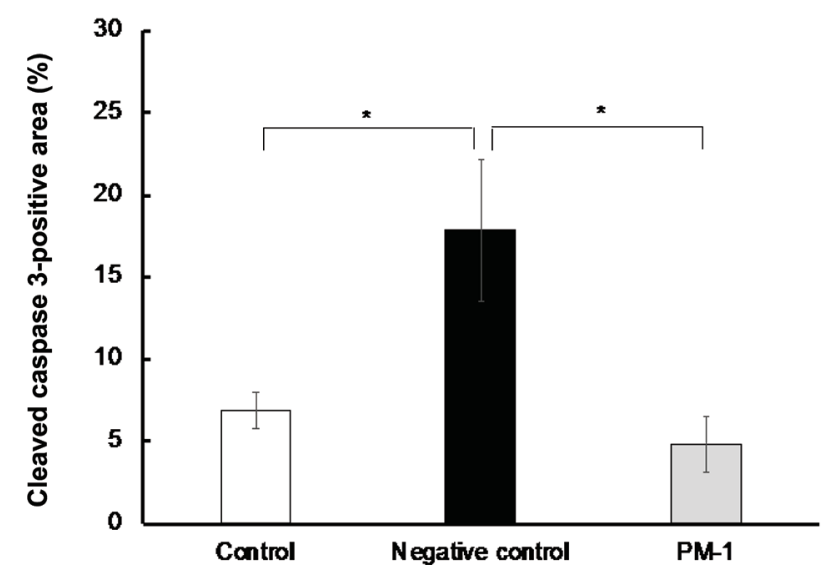

Figure 2. Immunohistochemistry analysis of cleaved caspase- 3 in the hippocampus of mice at $40 \times$ and $100 \times$ magnification (A; arrows show cleaved caspase-3-positive area), and the cleaved caspase-3-positive area $(\mathrm{B} ; \%)$. Values are the mean \pm SEM $(\mathrm{n}=4)$. $*$ Significantly different $(P<0.05)$ compared with the negative control group. Control, negative control, and probiotic mixture-1 (PM-1) treatments are as described in Table 1 .

nificantly reduced the expression of cleaved caspase-3 in hippocampus of mice, indicating a reduced level of injury in the hippocampus compared with mice in the negative control group $(P<0.05$, Figure 2$)$.

\section{Effect of PM-1 on Antioxidant Enzymes and MDA Production}

To demonstrate the underlying mechanism of the protective effect of PM-1 in D-galactose-treated aging mice, the plasma, brain, and liver of D-galactose-treated aging mice were evaluated for antioxidant enzyme activities and MDA production. Treatment with PM-1 significantly $(P<0.05)$ enhanced activities of antioxidant enzymes CAT $(29.5 \pm 6.2 \mathrm{U} / \mathrm{mL})$ and SOD (0.6 $\pm 0.1 \mathrm{U} / \mathrm{mL}$ ) in plasma compared with the negative control group (CAT: $12.5 \pm 5.4 \mathrm{U} / \mathrm{mL}$; SOD: $0.2 \pm 0.1$ $\mathrm{U} / \mathrm{mL}$ ). The production of MDA in plasma of mice of the negative control group $(11.6 \pm 0.9 \mu M)$ was higher than that in mice of the control group $(6.9 \pm 0.8 \mu M)$ (Figure 3C), whereas MDA production was decreased in mice of the PM-1 group $(7.7 \pm 0.8 \mu M ; P<0.05)$. Similar protective effects of PM-1 were found in brain and liver tissue in D-galactose-treated mice (Figure $3 \mathrm{D}-\mathrm{J})$. Thus, treatment of PM-1 could ameliorate oxidative stress in D-galactose-treated aging mice. 


\section{Effect of PM-1 on Antioxidant-Related Protein Expression}

We analyzed antioxidant-related protein expression of liver tissue lysates by Western blotting. The expression of Nrf2 and HO-1 was decreased in the liver of D-galactose-treated aging (negative control) mice compared with that of the control group. Expression of Nrf2 and HO-1 was enhanced after PM-1 treatment, however, indicating that administration of PM-1 might promote the liver antioxidant activity of D-galactosetreated mice (Figure 4).

\section{Effect of PM-1 on Short-Chain Fatty Acid Production in $D$-Galactose-Treated Aging Mice}

To investigate whether short-chain fatty acids (SCFA) play a role in the antiaging effect of PM-1, we analyzed fecal SCFA contents of mice from each group. Contents of the SCFA (acetic acid, propionic acid, and butyric acid) were increased in the negative control group compared with the control group (Figure 5). After administration of PM-1, SCFA contents were decreased compared with that in mice of the negative control group.

\section{Plasma}

(A)

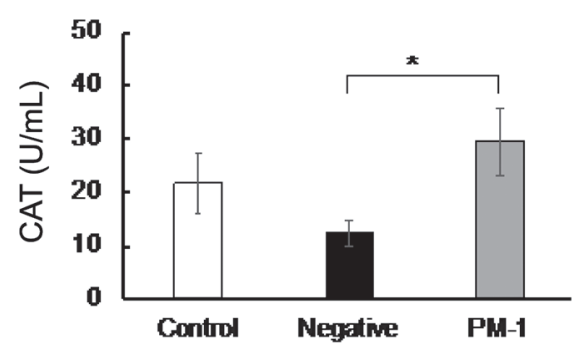

\section{Brain}

(D)

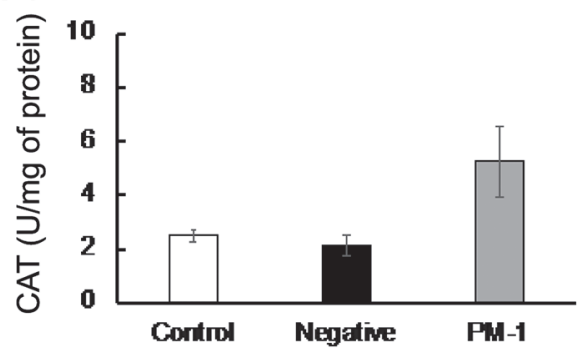

Liver

(G)

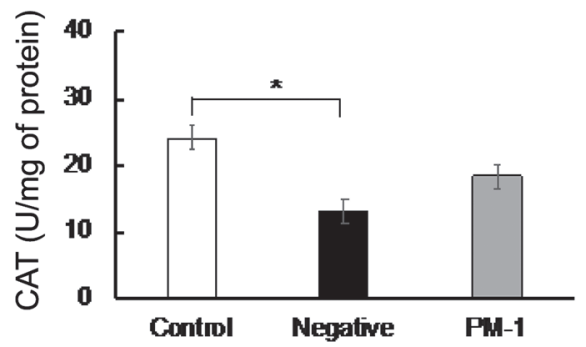

(B)

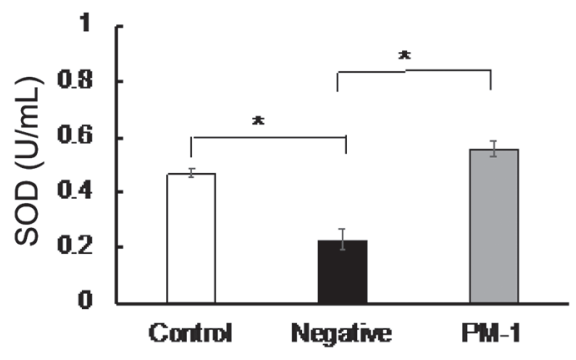

(E)

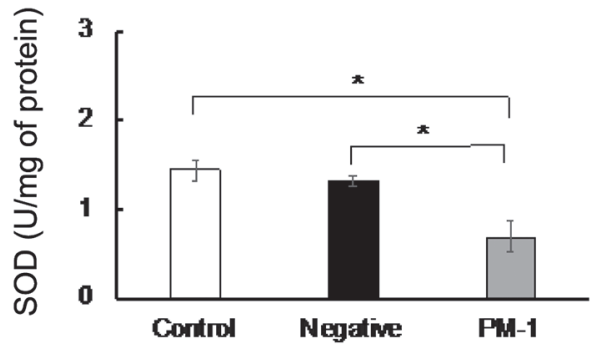

(H)

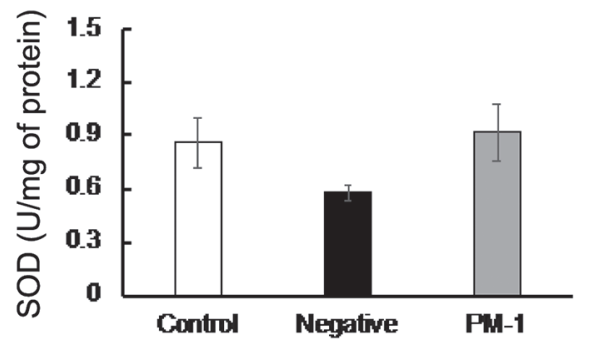

(C)

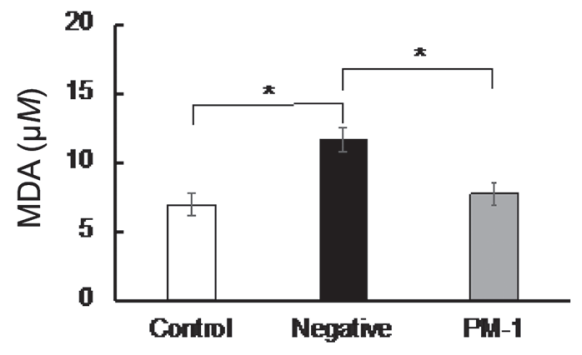

(F)

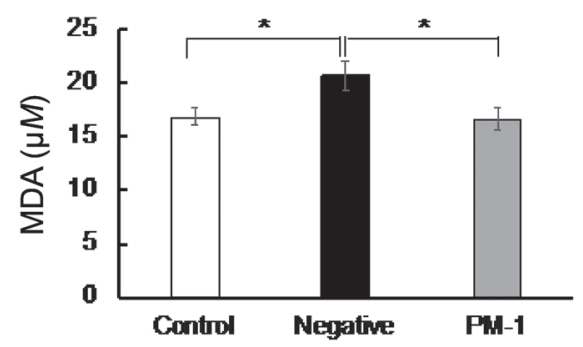

(I)

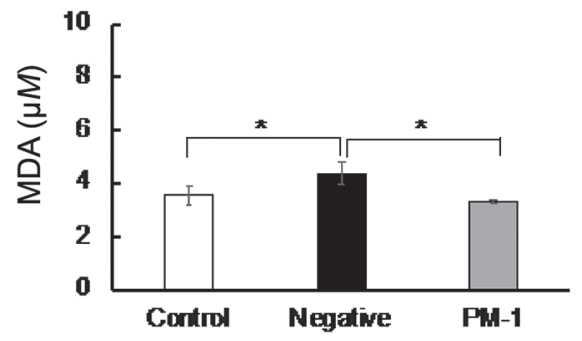

Figure 3. The in vivo effect of probiotic mixture-1 (PM-1) on catalase (CAT) activity (A, D, G), superoxide dismutase (SOD) activity (B, $\mathrm{E}, \mathrm{H})$, and malondialdehyde (MDA) production (C, F, I) in plasma, brain, and liver, respectively, of D-galactose-treated aging mice. Values are the mean \pm SEM $(\mathrm{n}=5)$. *Significantly different $(P<0.05)$ between treatments. Control, negative control, and PM-1 treatments are as described in Table 1. 
(A)

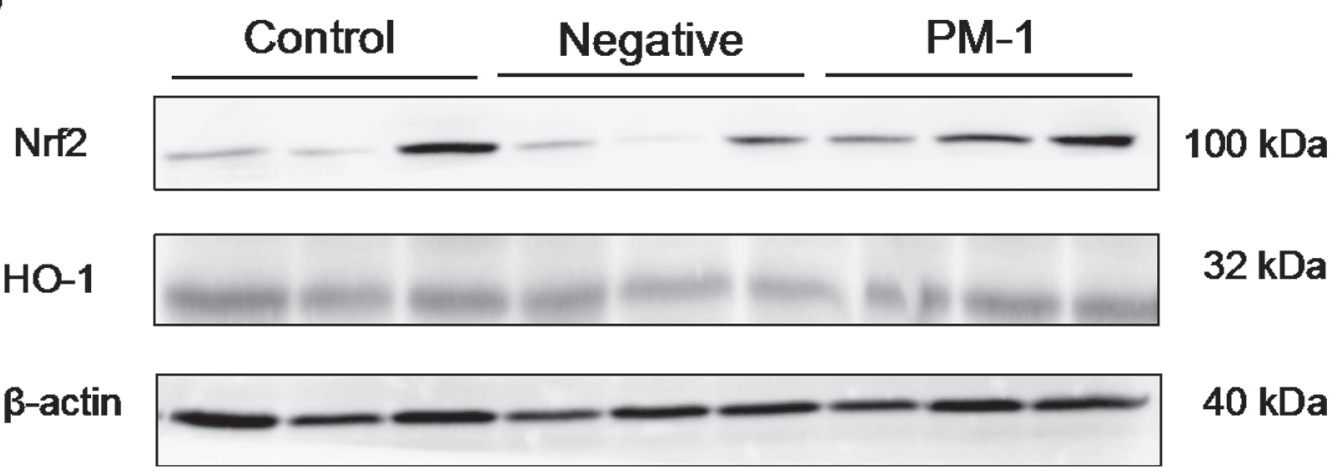

(B)

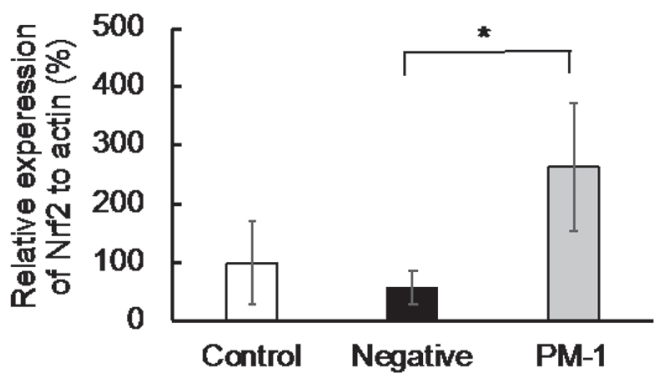

(C)

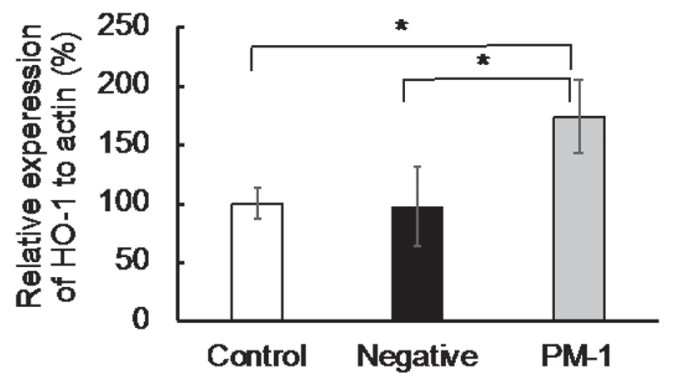

Figure 4. The in vivo effect of probiotic mixture-1 (PM-1) on expression of antioxidant-related proteins nuclear factor erythroid 2-related factor 2 (Nrf2), heme oxygenase-1 (HO-1), and $\beta$-actin (A), relative expression of Nrf2 (B), and relative expression of HO-1 (C) in the liver of D-galactose-treated aging mice. Values are the mean \pm SEM $(\mathrm{n}=3)$. *Significantly different $(P<0.05)$ between treatments. Control, negative control, and PM-1 treatments are as described in Table 1.

\section{DISCUSSION}

In the present study, we demonstrated that long-term (10 wk) administration of PM-1 could improve learning and memory abilities in a D-galactose-treated aging mouse model. Inhibition of cell apoptosis and brain injury was also observed following PM-1 administration. Using D-galactose to induce aging and brain injury in rodents is a well-known model to evaluate antiaging effects of test samples (Parameshwaran et al., 2010; Fu et al., 2017). The D-galactose-induced impairments of learning and memory extended escape latencies, decreased cumulative duration of target quadrant in treated mice in the Morris water maze test, and induced brain toxicity in mice. Although several studies have focused on the antiaging effect of probiotics (Yu et al., 2016; Zhao et al., 2017), few have shown the effects of a combination of probiotic strains on antiaging in rodent models and human pilot studies.

We hypothesized that the antiaging effect of PM-1 was related to the antioxidative effect by an alteration of the microbiota by PM-1, which contributed to change key metabolites - the SCFA. Our results highlight 3 interdependent effects on improving the abilities

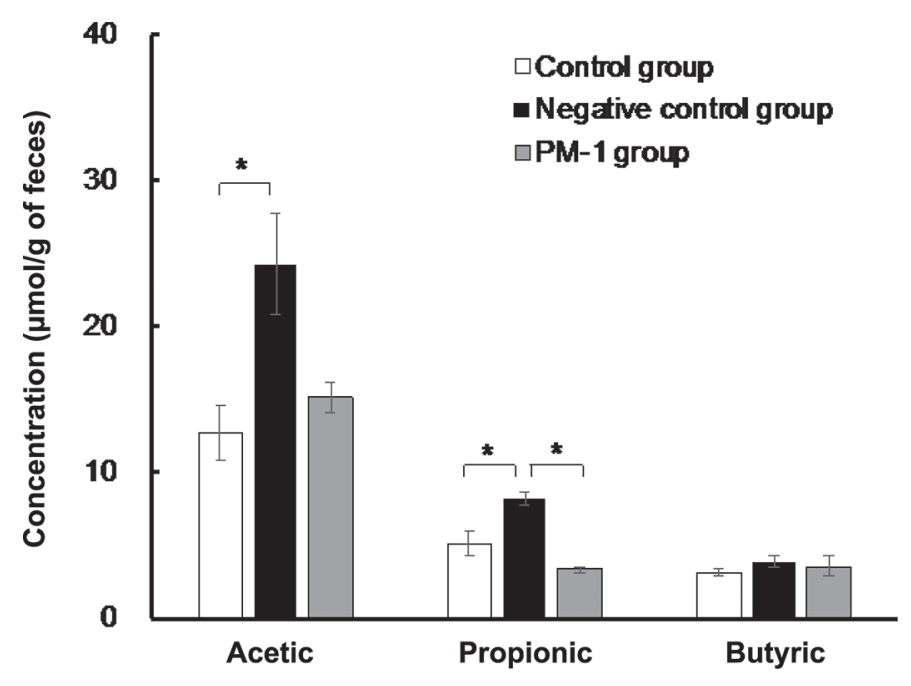

Figure 5. Analysis of fecal short-chain fatty acids (acetic, propionic, and butyric acids). Values are the mean \pm SEM $(n=5)$. * Significantly different $(P<0.05)$ between treatments. Control, negative control, and probiotic mixture-1 (PM-1) treatments are as described in Table 1. 
of learning and memory in a D-galactose-treated aging mice model through administration of PM-1.

First, we demonstrated that PM-1 could reduce activation of caspase-3 in the hippocampus in D-galactose-treated aging mice. This result implied that the improvement of memory and learning activities in mice might due to an antiapoptotic effect of PM-1. Previous studies reported that caspase- 3 is a critical apoptosis mediator involved in both intrinsic and extrinsic apoptosis pathways, leading to brain injury after activation by ROS and other factors (Jang et al., 2002; Schon and Manfredi, 2003; Lan et al., 2012).

Second, administration of PM-1 enhanced the antioxidant defense system and restored the memory and learning abilities of D-galactose-treated aging mice by enhancing antioxidant enzyme activity (SOD and CAT) in plasma. These 2 antioxidant enzymes contribute to the antioxidant defense system by eliminating ROS and then protecting cells and tissues from cellular oxidative damage. Moreover, SOD can convert superoxide radical, a primary ROS produced by the electron transport chain in the mitochondria, to hydrogen peroxide, which is further broken down into water by CAT or glutathione peroxidase. Hydrogen peroxide needs to be removed immediately or it will generate more active radicals through the Fenton reaction (Paradies et al., 2011). Lipid peroxidation, a factor related to oxidative stress, is also closely associated with the aging process and aging-related diseases (Spiteller, 2001; Praticò, 2002). Decreased production of MDA, an indicator of oxidative damage by lipid peroxidation, also indicated that PM-1 could protect brain damage by inhibiting lipid peroxidation.

Additionally, the upstream regulatory factors in Dgalactose-treated aging mice demonstrated that PM-1 treatment could restore Nrf2 and HO-1 expression in the liver of D-galactose-treated aging mice. A multifunctional regulator, Nrf2 plays a vital role in regulating the expression of antioxidant, anti-inflammatory, and detoxifying proteins. The major functions of Nrf2 are maintaining cellular redox status balance, promoting antioxidant activity, and inducing the phase II detoxification pathway in mammals (Loboda et al., 2008; Chen et al., 2016b). Heme oxygenase-1, one of the downstream target genes regulated through Nrf2, catalyzes the oxidative degradation of heme, resulting in generation of $\mathrm{CO}$, iron ions, and biliverdin (Loboda et al., 2008, 2016). Thus, Nrf2, HO-1, and other antioxidant enzymes contribute to protection from oxidative stress, cell death, inflammatory response, and angiogenesis (Loboda et al., 2016). Thus, a possible antioxidant pathway is that PM-1 activates the antioxidant enzymes SOD and CAT by inducing Nrf2 or HO-1 expression in D-galactose-treated aging mice.

Third, fecal SCFA concentrations of mice were elevated after treatment with D-galactose, whereas PM-1 administration decreased the fecal SCFA concentration. Short-chain fatty acids are reported to have many health-beneficial effects (LeBlanc et al., 2017; Ohira et al., 2017). However, excess SCFA concentrations in the body can cause an inflammatory response and metabolic dysfunction (Ohira et al., 2017). For example, excessive propionate will cause oxidative stress, neurotoxicity, propionic acidemia, and neurological impairment in vivo (Fontella et al., 2000; Khalil et al., 2015). In addition to the in vivo effect, Jan et al. (2002) reported that elevated concentrations of propionate induced apoptosis and generated ROS in human colorectal carcinoma cell lines. The adverse effects of acetate and butyrate have also been reported in previous studies (Peng et al., 2007; Park et al., 2016). Because of the close association between SCFA production and intestinal microbes, we speculated that intestinal microbes were also a regulatory factor for the antiaging effect of PM-1. The intestinal microbiota can be modulated by many factors, including environmental factors, diet, and additional probiotic supplements. Our unpublished data reveal that administration of PM-1 enhances the relative abundance of Bifidobacterium and Lactobacillus species and decrease that of the Enterobacteriaceae in the ceca of mice. Thus, we surmised that administration of PM-1 could restore the SCFA concentration to an appropriate level by modulating the intestinal microbiota; however, the exact mechanism remains unclear and further investigation is required.

\section{CONCLUSIONS}

Our study provides an example of how probiotics can act as a potential therapy for aging-related dysfunction. Our results indicate that PM-1-induced modulation of SCFA might regulate antioxidant enzymes by inducing expression of Nrf2 or HO-1, and that administration of PM-1 inhibits cell apoptosis and brain injury, resulting in improved memory and learning abilities in D-galactose-treated aging mice. Further studies are warranted to examine the potential clinical use of PM-1 as a novel therapeutic in ameliorating the aging process.

\section{ACKNOWLEDGMENTS}

This work was supported by the project 106AS3.5.2AD-U1 of the Council of Agriculture, Executive 
Yuan (Taipei, Taiwan). The authors declare no conflicts of interest.

\section{REFERENCES}

Alam, M. N., N. J. Bristi, and M. Rafiquzzaman. 2013. Review on in vivo and in vitro methods evaluation of antioxidant activity. Saudi Pharm. J. 21:143-152.

Barja, G. 2014. The mitochondrial free radical theory of aging. Prog. Mol. Biol. Transl. Sci. 127:1-27.

Barzilai, N., D. M. Huffman, R. H. Muzumdar, and A. Bartke. 2012. The critical role of metabolic pathways in aging. Diabetes 61:13151322.

Chen, C. L., K. Y. Chang, and T. M. Pan. 2016a. Monascus purpureus NTU 568 fermented product improves memory and learning ability in rats with aluminium-induced Alzheimer's disease. J. Funct. Foods 21:167-177.

Chen, P., D. He, Y. Zhang, S. Yang, L. Chen, S. Wang, H. Zou, Z. Liao, X. Zhang, and M. Wu. 2016b. Sargassum fusiforme polysaccharides activate antioxidant defense by promoting Nrf2-dependent cytoprotection and ameliorate stress insult during aging. Food Funct. 7:4576-4588.

Chen, Z., Y. Tang, A. Liu, X. Jin, J. Zhu, and X. Lu. 2017. Oral administration of Grifola frondosa polysaccharides improves memory impairment in aged rats via antioxidant action. Mol. Nutr. Food Res. 61. https://doi.org/10.1002/mnfr.201700313.

Dillin, A., D. E. Gottschling, and T. Nyström. 2014. The good and the bad of being connected: The integrons of aging. Curr. Opin. Cell Biol. 26:107-112.

Fontella, F. U., V. Pulrolnik, E. Gassen, C. M. Wannmacher, A. B. Klein, M. Wajner, and C. S. Dutra-Filho. 2000. Propionic and Lmethylmalonic acids induce oxidative stress in brain of young rats. Neuroreport 11:541-544.

Fu, W., H. Wang, X. Ren, H. Yu, Y. Lei, and Q. Chen. 2017. Neuroprotective effect of three caffeic acid derivatives via ameliorate oxidative stress and enhance PKA/CREB signaling pathway. Behav. Brain Res. 328:81-86.

Harman, D. 2002. Alzheimer's disease: Role of aging in pathogenesis. Ann. N. Y. Acad. Sci. 959:384-395.

Jan, G., A. S. Belzacq, D. Haouzi, A. Rouault, D. Métivier, G. Kroemer, and C. Brenner. 2002. Propionibacteria induce apoptosis of colorectal carcinoma cells via short-chain fatty acids acting on mitochondria. Cell Death Differ. 9:179-188.

Jang, M. H., M. C. Shin, H. S. Shin, K. H. Kim, H. J. Park, E. H. Kim, and C. J. Kim. 2002. Alcohol induces apoptosis in TM3 mouse Leydig cells via Bax-dependent caspase-3 activation. Eur. J. Pharmacol. 449:39-45.

Khalil, S. R., Y. M. Abd-Elhakim, M. E. Selim, and L. Y. A. AlAyadhi. 2015. Pitoxin protects rat pups brain from propionic acidinduced oxidative stress: The expression pattern of Bcl-2 and Caspase-3 apoptotic genes. Neurotoxicology 49:121-131.

Lan, Y. H., J. H. Chiang, W. W. Huang, C. C. Lu, J. G. Chung, T. S. Wu, J. H. Jhan, K. L. Lin, S. J. Pai, Y. J. Chiu, M. Tsuzuki, and J. S. Yang. 2012. Activations of both extrinsic and intrinsic pathways in HCT 116 human colorectal cancer cells contribute to apoptosis through p53-mediated ATM/Fas signaling by Emilia sonchifolia extract, a folklore medicinal plant. Evid. Based Complement. Alternat. Med. 2012:178178.

LeBlanc, J. G., F. Chain, R. Martín, L. G. Bermúdez-Humarán, S. Courau, and P. Langella. 2017. Beneficial effects on host energy metabolism of short-chain fatty acids and vitamins produced by commensal and probiotic bacteria. Microb. Cell Fact. 16:79.

Li, S., Y. Zhao, L. Zhang, X. Zhang, L. Huang, D. Li, and Q. Wang. 2012. Antioxidant activity of Lactobacillus plantarum strains isolated from traditional Chinese fermented foods. Food Chem. 135:1914-1919.

Loboda, A., M. Damulewicz, E. Pyza, A. Jozkowicz, and J. Dulak. 2016. Role of Nrf2/HO-1 system in development, oxidative stress response and diseases: An evolutionarily conserved mechanism. Cell. Mol. Life Sci. 73:3221-3247.

Loboda, A., A. Jazwa, A. Grochot-Przeczek, A. J. Rutkowski, J. Cisowski, A. Agarwal, A. Jozkowicz, and J. Dulak. 2008. Heme oxygenase-1 and the vascular bed: From molecular mechanisms to therapeutic opportunities. Antioxid. Redox Signal. 10:1767-1812.

Ohira, H., W. Tsutsui, and Y. Fujioka. 2017. Are short chain fatty acids in gut microbiota defensive players for inflammation and atherosclerosis? J. Atheroscler. Thromb. 24:660-672.

Paradies, G., G. Petrosillo, V. Paradies, and F. M. Ruggiero. 2011. Mitochondrial dysfunction in brain aging: Role of oxidative stress and cardiolipin. Neurochem. Int. 58:447-457.

Parameshwaran, K., M. H. Irwin, K. Steliou, and C. A. Pinkert. 2010. D-Galactose effectiveness in modeling aging and therapeutic antioxidant treatment in mice. Rejuvenation Res. 13:729-735.

Park, J., C. J. Goergen, H. HogenEsch, and C. H. Kim. 2016. Chronically elevated levels of short-chain Fatty acids induce T cell-mediated ureteritis and hydronephrosis. J. Immunol. 196:2388-2400.

Peng, L., Z. He, W. Chen, I. R. Holzman, and J. Lin. 2007. Effects of butyrate on intestinal barrier function in a Caco-2 cell monolayer model of intestinal barrier. Pediatr. Res. 61:37-41.

Praticò, D. 2002. Lipid peroxidation and the aging process. Sci. Aging Knowledge Environ. 2002:re5.

Qu, Z., J. Zhang, H. Yang, L. Huo, J. Gao, H. Chen, and W. Gao. 2016. Protective effect of tetrahydropalmatine against d-galactose induced memory impairment in rat. Physiol. Behav. 154:114-125.

Raz, N., and A. M. Daugherty. 2018. Pathways to brain aging and their modifiers: Free-radical-induced energetic and neural decline in senescence (FRIENDS) model-A mini-review. Gerontology 64:49-57.

Rottenberg, H., and J. B. Hoek. 2017. The path from mitochondrial ROS to aging runs through the mitochondrial permeability transition pore. Aging Cell 16:943-955.

Schon, E. A., and G. Manfredi. 2003. Neuronal degeneration and mitochondrial dysfunction. J. Clin. Invest. 111:303-312.

Spiteller, G. 2001. Lipid peroxidation in aging and age-dependent diseases. Exp. Gerontol. 36:1425-1457.

Tang, W., Z. Xing, W. Hu, C. Li, J. Wang, and Y. Wang. 2016. Antioxidative effects in vivo and colonization of Lactobacillus plantarum MA2 in the murine intestinal tract. Appl. Microbiol. Biotechnol. 100:7193-7202

Torii, T., K. Kanemitsu, T. Wada, S. Itoh, K. Kinugawa, and A. Hagiwara. 2010. Measurement of short-chain fatty acids in human faeces using high-performance liquid chromatography: specimen stability. Ann. Clin. Biochem. 47:447-452.

Ward, R. J., F. A. Zucca, J. H. Duyn, R. R. Crichton, and L. Zecca 2014. The role of iron in brain ageing and neurodegenerative disorders. Lancet Neurol. 13:1045-1060.

Yu, X., S. J. Li, D. Yang, L. Qiu, Y. P. Wu, D. Y. Wang, and H. Wei. 2016. A novel strain of Lactobacillus mucosae isolated from a Gaotian villager improves in vitro and in vivo antioxidant as well as biological properties in D-galactose-induced aging mice. J. Dairy Sci. 99:903-914.

Zhao, J., F. Tien, N. Zhao, Q. Zhai, H. Zhang, and W. Chen. 2017. Effects of probiotics on D-galactose-induced oxidative stress in plasma: A meta-analysis of animal models. J. Funct. Foods 39:44-49.

Zhong, S. Z., Q. H. Ge, R. Qu, Q. Li, and S. P. Ma. 2009. Paeonol attenuates neurotoxicity and ameliorates cognitive impairment induced by D-galactose in ICR mice. J. Neurol. Sci. 277:58-64. 